\title{
Frequência de sofrimento emocional é elevada em pessoas com diabetes assistidas na atenção primária
}

\section{Frequency of emotional distress is high in people with primary care-assisted diabetes \\ La frecuencia de sufrimiento emocional es elevada en personas con diabetes que reciben asistencia en la atención primaria}

\author{
Juliana Andrade Goes ${ }^{1} \odot$, Karla Ferreira Rodrigues ${ }^{2} \odot$, Ana Carolina de Avila ${ }^{1} \oplus$, Aline Geisler ${ }^{1} \oplus$, Amanda Maieski ${ }^{1} \bullet$, \\ Carlos Roberto de Oliveira Nunes ${ }^{2} \oplus$, Joao Luiz Gurgel Calvet da Silveira² $\odot$, Ernani Tiaraju de Santa Helena² \\ ${ }^{1}$ Curso de Medicina da Universidade Regional de Blumenau - FURB-Blumenau, Santa Catarina, Brasil. \\ ${ }^{2}$ Programa de Pós-Graduação em Saúde Coletiva da Universidade Regional de Blumenau - FURB-Blumenau, Santa Catarina, Brasil.
}

\section{Resumo}

Introdução: Pessoas com diabetes podem sofrer com o estresse da doença e apresentar sentimentos como culpa, raiva, medo e depressão, que caracterizam o Sofrimento Emocional Específico da Diabetes. Objetivo: estimar a frequência desse sofrimento e seus fatores associados em pessoas assistidas na atenção primária em Blumenau, Santa Catarina. Métodos: Trata-se de estudo transversal. Pessoas com diabetes assistidas por 4 equipes de saúde da família $(\mathrm{n}=196)$ responderam ao questionário "Problems Areas in Diabetes", que apresenta 20 questões em 4 subdimensões, além de questões sobre suas características sociodemográficas (sexo, idade, escolaridade) e clínicas (tempo de doença, uso de insulina e medicação antidepressiva). Estimou-se os escores de sofrimento geral e subdimensões com base na soma das respostas em escala de 0 (melhor) a 100 (pior). Mediu-se a frequência do sofrimento emocional grave (escore $>40$ ) e sua associação com as variáveis de estudo por regressão logística não condicional. Resultados: Participaram 196 pessoas, 58,2\% eram mulheres, 26,2\% faziam uso de insulina e $20,6 \%$ de antidepressivos. A idade média foi de 61,6 anos, o tempo médio de tratamento de diabetes foi 9,5 anos. O escore médio de sofrimento emocional foi de $33,6(\mathrm{dp}=27,6)$ e mediana de 23,8 . $36,2 \%$ dos participantes apresentaram sofrimento emocional grave. O sofrimento emocional grave se mostrou principalmente entre pessoas com 19 a 64 anos (OR=2,1, IC95\%1,1 - 4,1), com tempo de doença de 2 a 5 anos (OR=6,4; IC95\% 1,1 - 36,1) e 5 anos e mais (OR=5,4; IC95\% 1,1 - 28,8) e em uso de medicação antidepressiva (OR=2,8 IC95\% 1,3 - 6,0). Conclusão: Mais de um terço das pessoas com diabetes tem sofrimento emocional grave, marcadamente os adultos com mais tempo de doença e com tratamento para depressão. Sugere-se que essas pessoas tenham seu cuidado priorizado pelas equipes de saúde na atenção primária.

Palavras-chave: Diabetes Mellitus; Estresse Psicológico; Atenção Primária à Saúde; Qualidade de Vida

Como citar: Goes JA, Rodrigues KF, Avila AC, Geisler A, Maieski A, Nunes CRO, Silveira JLGC, Helena ETS. Frequência de sofrimento emocional é elevada em pessoas com diabetes assistidas na atenção primária. Rev Bras Med Fam Comunidade. 2020;15(42):2078. https://doi.org/10.5712/rbmfc15(42)2078

\author{
Autor correspondente: \\ Autor. Juliana Andrade Goes \\ E-mail: juliana.andradegoes@gmail.com \\ Fonte de financiamento: \\ Programa PET-Saúde do Ministério da \\ Saúde. \\ Parecer CEP: \\ Parecer 435.159 do CEP-FURB. \\ Procedência e revisão por pares: \\ Não encomendado; \\ revisão por pares externa. \\ Recebido em: 05/07/2019. \\ Aprovado em: 08/09/2019.
}




\begin{abstract}
Introduction: Diabetic persons can suffer from stress of the disease and present feelings such as guilt, anger, fear and depression, featuring the diabetes specific-emotional distress. Objective: to measure the frequency of this distress and its associated factors in people assisted in the primary health care of Blumenau, Santa Catarina. Methods: This is a cross-sectional study. People with diabetes assisted by 4 family health teams $(n=196)$ answered the "Problems Areas in Diabetes" questionnaire, which presents 20 questions in 4 sub-dimensions, as well as questions about their sociodemographic (gender, age, education) and clinical characteristics (insulin and antidepressant medication use). Overall distress scores and sub-dimensions were estimated based on the sum of responses on a scale from 0 (best) to 100 (worst). The frequency of severe emotional distress was measured (score> 40) and its association with study variables were estimated by unconditional logistic regression. Results: 196 people participated, $58.2 \%$ were women, $26.2 \%$ were on insulin and $20.6 \%$ on antidepressants. The mean age was 61.6 years and the mean duration of diabetes treatment was 9.5 years. The average score of emotional distress was $33.6(\mathrm{dp}=27,6)$ and median of $23,8.36,2 \%$ of participants had severe emotional distress. Severe emotional distress was mainly seen among people aged $19-64$ years $(\mathrm{OR}=2.1,95 \% \mathrm{Cl} 1.1-4.1)$, with disease duration from 2 to 5 years $(\mathrm{OR}=6.4,95 \% \mathrm{Cl}, 1-36.1)$ and 5 years andmore $(\mathrm{OR}=5.4,95 \% \mathrm{Cl} 1.1-28.8)$ and on antidepressant medication $(\mathrm{OR}=2.895 \%$ $\mathrm{Cl}$ 1.3 - 6.0). Conclusion: More than a third of people with diabetes have severe emotional distress, notably adults with longer ilness and treatment for depression. It is suggested that these people must have their care prioritized by health teams in primary care.
\end{abstract}

Keywords: Diabetes Mellitus; Stress, Psychological; Primary Health Care; Quality of Life

\title{
Resumen
}

Introducción: Las personas con diabetes pueden sufrir con el estrés de la enfermedad y presentar sentimientos como culpa, rabia, miedo y depresión, que caracterizan el Sufrimiento Emocional Específico de la Diabetes. Objetivo: estimar la frecuencia de ese sufrimiento y factores asociados en personas asistidas en la atención primaria en Blumenau, Santa Catarina. Métodos: este es un estudio transversal. Las personas con diabetes asistidas por 4 equipos de salud de la familia $(n=196)$ respondieron el cuestionario "Áreas problemáticas en diabetes", que presenta 20 preguntas en 4 subdimensiones, así como preguntas sobre sus características sociodemográficas (género, edad, educación) y características clínicas (tiempo de enfermedad, uso de insulina y medicación antidepresiva). Los puntajes de angustia y subdimensiones generales se estimaron en función de la suma de las respuestas en una escala de 0 (mejor) a 100 (peor). Se ha medido la frecuencia de angustia emocional severa (puntaje $>40$ ) y su asociación con variables de estudio por regresión logística incondicional. Resultados: Han participado 196 personas, 58,2\% eran mujeres, 26,2\% hacían uso de insulina y $20,6 \%$ de antidepresivos. La edad media fue de 61,6 años, el tiempo promedio de tratamiento de la diabetes fue de 9,5 años. El puntaje promedio de sufrimiento emocional fue de $33,6(\mathrm{dp}=27,6)$ y mediana de 23,8 . El $36,2 \%$ de los participantes presentaron un sufrimiento emocional grave. El sufrimiento emocional grave se mostró principalmente entre personas de 19 a 64 años $(O R=2,1$, IC95\% 1,1 - 4,1), con tiempo de enfermedad de 2 a 5 años (OR = 6,4, IC95\% 1,1 - 36,1) y 5 años y más $(O R=5,4$, IC95\% 1,1 - 28,8) y en uso de medicación antidepresiva $(\mathrm{OR}=2,8 \mathrm{IC95 \%} 1,3-6,0)$. Conclusión: Más de un tercio de las personas con diabetes tienen sufrimiento emocional severa, especialmente adultos con enfermedades más prolongadas y tratamiento para la depresión. Se sugiere que estas personas tengan su atención priorizada por los equipos de salud en la atención primaria.

Palabras clave: Diabetes Mellitus; Estrés Psicológico; Atención Primaria de Salud; Calidad de Vida

\section{Introdução}

Diabetes mellitus (DM) é uma condição crônica caracterizada pela hiperglicemia. Estudos de diabetes autorreferida estimaram valores entre 6,2 e 11,7\% em adultos, chegando a 22,9\% na população com mais de 65 anos, ${ }^{1,2}$ o que representa um problema crescente de saúde pública no Brasil. Pessoas que vivem com o diabetes podem apresentar sofrimento mental com a doença, ${ }^{3,4}$ pois demanda ajustes nas rotinas dos pacientes que envolvem controle metabólico, monitoramento regular da glicemia, uso contínuo de medicamentos, dietas balanceadas e prática regular de exercícios físicos. Além disso, muitos pacientes se preocupam com o desenvolvimento de complicações relacionadas à doença. ${ }^{5,6}$

As pessoas que vivem com a diabetes podem apresentar sentimentos negativos que elas associam ao fato de viverem com essa doença, o que caracteriza a condição chamada de Sofrimento Mental Específico da Diabetes (SMED). ${ }^{6}$ Estes sentimentos podem envolver ansiedade, culpa, humor depressivo, medo e preocupação. $^{4}$ 
Essa condição específica, frequentemente não identificada, dificulta o controle da doença e está relacionada a uma piora no controle glicêmico. ${ }^{5,7,8}$ Uma revisão sistemática com metanálise que incluiu 55 estudos ( $n=36.998$ ) encontrou uma prevalência ponderada de $36 \%$ de SMED em pessoas com DM tipo 2. Esses valores eram ainda maiores em pessoas do sexo feminino e com sintomas depressivos. ${ }^{9}$ Essa condição tem sido identificada a partir de diferentes instrumentos testados e validados em diversos países, entre os quais se destacam o Problems Area in Diabetes Scale (PAID) e o Diabetes Distress Scale (DDS). ${ }^{6,10-13}$

Os poucos estudos nacionais acerca desse tema enfocam sua relação com a qualidade de vida e se dão em ambiente hospitalar ou em serviço especializado. ${ }^{14-16}$ De outro lado, o modelo de organização preconizado pelo Sistema Único de Saúde (SUS) considera que a integralidade do cuidado à pessoa com diabetes deve ser coordenado pela atenção primária. ${ }^{17}$

Blumenau, município da Região Sul do Brasil, no ano de 2013 tinha uma população estimada de 329.082 habitantes, com um Índice de Desenvolvimento Humano Municipal de 0,806 e expectativa de vida de 78,64 anos. ${ }^{18}$ Nesse mesmo ano, a cobertura de equipes da atenção primária era de $89,1 \%$, na qual estavam cadastradas 5161 pessoas com DM no "Sistema de Gestão Clínica de Hipertensão Arterial e Diabetes Mellitus da Atenção Básica” (SISHIPERDIA). ${ }^{19}$

O objetivo dessa pesquisa foi identificar o SMED autorreferido das pessoas com diabetes assistidas na atenção primária em Blumenau, SC, e seus possíveis fatores associados.

\section{Métodos}

Trata-se de um estudo quantitativo observacional e transversal. A população de estudo consistiu em 244 pessoas com DM de 4 unidades básicas de saúde de Blumenau, cadastradas no SISHIPERDIA. Essas unidades foram escolhidas porque eram cenários de projetos de pesquisa e extensão da Universidade Regional de Blumenau em parceria com a Secretaria Municipal de Saúde, com o apoio do Programa de Educação para o Trabalho (PET-Saúde) do Ministério da Saúde.

Para serem incluídas, as pessoas deveriam ter mais de 18 anos, diagnóstico confirmado de $\mathrm{DM}^{20} \mathrm{e}$ assinar o Termo de Consentimento Livre e Esclarecido. Foram excluídos aqueles que por motivo físico ou cognitivo não apresentassem condições de responder às perguntas.

A coleta de dados se deu entre novembro de 2013 e março de 2014. Todas as pessoas com diabetes foram contatadas por telefone (até três tentativas) e por visita domiciliar (até duas tentativas). Aquelas que foram contatadas e que concordaram em participar foram entrevistadas em domicílio por um estudantebolsista do PET-Saúde. Os estudantes foram previamente treinados para a aplicação dos questionários mediante a leitura do manual de aplicação, simulação de entrevistas, resolução de dúvidas com supervisores em dois encontros presenciais e aplicação de teste piloto com pessoas que não participaram da amostra final do estudo.

Foram coletadas as seguintes variáveis de estudo: sexo, idade (em anos completos), escolaridade (analfabeto/escreve o nome, ensino fundamental/médio, ensino superior), se mora sozinho ou não, se tem parceiro ou não, consumo atual de tabaco, tempo de diagnóstico de diabetes (em anos), uso de insulina (em caso afirmativo, há quanto tempo) e uso de medicação antidepressiva. 
A variável de desfecho foi o Sofrimento Mental Específico da Diabetes, estimado através do questionário PAID. Inicialmente validado em pacientes com diabetes tipo 1, mostrou elevada consistência interna (alfa de Cronbach=0,95). Apesar de não existir padrão-ouro, o instrumento se associou positivamente a medidas psicossociais como angústia emocional geral, transtornos alimentares, medo da hipoglicemia, complicações diabéticas de curto e longo prazo e hemoglobina glicosilada alterada, e negativamente associada a comportamentos de autocuidado relatado. ${ }^{2} \mathrm{~A}$ versão traduzida e validada para língua portuguesa, predominantemente com pessoas com diabetes tipo 2 , mostrou condições psicométricas e de validade adequadas (alfa de Cronbach=0,93). ${ }^{21}$

O questionário apresenta 20 questões divididas em 4 subdimensões: problemas emocionais relacionados ao diabetes (12 questões); problemas relacionados ao tratamento (3 questões); problemas relacionados à alimentação (3 questões); problemas relacionados ao apoio social (2 questões). Cada questão possui as opções de resposta: "Não é um problema", "Pequeno problema", "Problema moderado", "Quase um problema sério" e "Problema sério", que correspondem aos respectivos valores de 0, 1, 2, 3 e 4. Para se obter o escore total, somam-se as respostas dadas de 0 a 4 nas 20 questões do PAID, e multiplica-se por 1,25, de forma a produzir um escore que varia de 0 a 100. Quanto maior o escore atingido, maior o nível de sofrimento. Uma melhor avaliação pode ser realizada quando são analisados os escores nas diferentes subdimensões, correlacionando-os com as características dos participantes. Considera-se a pontuação 40 como valor corte para o PAID a fim de determinar se o paciente apresenta um sofrimento mental grave..$^{22}$

Os dados foram analisados por meio de estatística descritiva e as características dos participantes foram apresentadas por frequências absolutas e relativas (com respectivos intervalos de 95\% de confiança) em forma tabular. Para as variáveis contínuas, foram calculadas as medidas de tendência central (média e mediana) e de dispersão (desvio padrão [dp] e intervalos interquartílicos [IQ]).

Estimou-se o escore médio geral de SMED, suas subdimensões e a prevalência de sofrimento mental grave. Compararam-se os valores médios do escore total e de suas subdimensões e os valores agrupados de cada variável de estudo através do teste de Mann-Whittney ou do teste de correlação de Spearman para variáveis quantitativas (idade, tempo de doença e tempo de insulinoterapia), pois estas variáveis não apresentaram distribuição normal.

A possível associação entre a variável sofrimento emocional grave (variável dicotômica) e as variáveis de estudo foi estimada através do teste de Qui-quadrado. Aquelas variáveis que apresentaram significância de $p<0,20$ foram incluídas no modelo de regressão logística não condicional através do método Stepwise Backward. O modelo final, ajustado por sexo e faixa etária, foi testado através do método Goodness-of-fit de Hosmer-Lemeshow. Foi aceito um nível de significância de $p<0,05$.

O projeto de pesquisa foi aprovado pelo Comitê de Ética em Pesquisa da Universidade Regional de Blumenau (parecer 435.159).

\section{Resultados}

Dos 244 sujeitos com DM cadastrados, $16(6,6 \%)$ se recusaram a participar e $32(13,1 \%)$ não foram encontrados, de forma que participaram efetivamente 196 pessoas. 
A Tabela 1 mostra que a idade média dos participantes foi de 61,6 anos $(d p=13,1)$, com mediana de 63. Dos 196 participantes, 84 (42,9\%) tinham menos de 65 anos e 112 (57,1\%), 65 anos ou mais. O tempo médio de tratamento de diabetes foi 9,5 anos $(d p=7,6)$, a mediana de tempo foi de $8(I Q=3 ; 14)$ e amplitude de 39.

Com relação aos escores do PAID, a média total foi de 33,6 (dp=27,6), mediana de 23,8 (IQ=10;56,3) e amplitude de 97,5. Com relação às subdimensões do PAID, observou-se um valor médio de problemas emocionais de 22,0 ( $d p=18,6)$, de problemas com tratamento de 4,5 $(d p=4,3)$, problemas com alimentação $5,2(d p=4,9)$ e problemas relativos a apoio social 1,9 $(\mathrm{dp}=3,1)$. Apresentaram possível SMED grave 71 pessoas $(36,2 \%$, IC 95\% 29,5 - 43,4).

A Tabela 2 apresenta a associação entre os escores do PAID e suas subdimensões e as demais características das pessoas com diabetes.

Tabela 1. Características das pessoas com DM assistidas na APS em Blumenau, SC.

\begin{tabular}{|c|c|c|}
\hline Variáveis & Frequência absoluta & Frequência relativa (\%) \\
\hline \multicolumn{3}{|l|}{ Sexo $(n=196)$} \\
\hline Masculino & 82 & 41,8 \\
\hline Feminino & 114 & 58,2 \\
\hline \multicolumn{3}{|l|}{ Escolaridade $(n=195)$} \\
\hline Não alfabetizado ou sem declaração & 8 & 4,10 \\
\hline Ensino fundamental completo ou incompleto & 150 & 76,92 \\
\hline Ensino superior, mestrado ou doutorado & 37 & 18,97 \\
\hline \multicolumn{3}{|l|}{ Mora sozinho $(n=193)$} \\
\hline Sim & 23 & 11,9 \\
\hline Não & 170 & 88,1 \\
\hline \multicolumn{3}{|l|}{ Parceiro $(n=193)$} \\
\hline Sim & 143 & 74,1 \\
\hline Não & 50 & 25,9 \\
\hline \multicolumn{3}{|l|}{ Tempo de doença $(n=194)$} \\
\hline $0-15$ & 62 & 31,96 \\
\hline $5-110$ & 48 & 24,74 \\
\hline 10 e mais & 84 & 43,30 \\
\hline \multicolumn{3}{|l|}{ Insulina $(\mathrm{n}=195)$} \\
\hline Sim & 51 & 26,2 \\
\hline Não & 144 & 73,8 \\
\hline \multicolumn{3}{|l|}{ Tempo de insulinoterapia em anos $(n=194)$} \\
\hline 0 & 144 & 74,2 \\
\hline $0,1-12$ & 9 & 4,7 \\
\hline 2 e mais & 41 & 21,1 \\
\hline \multicolumn{3}{|l|}{ Usa medicação antidepressiva $(n=194)$} \\
\hline Sim & 40 & 20,6 \\
\hline Não & 154 & 79,4 \\
\hline \multicolumn{3}{|l|}{ Tabagismo $(n=195)$} \\
\hline Sim & 24 & 12,3 \\
\hline Não & 171 & 87,7 \\
\hline
\end{tabular}


Tabela 2. Médias dos escores totais do PAID e subdimensões associadas às características da amostra.

\begin{tabular}{|c|c|c|c|c|c|}
\hline \multirow{2}{*}{ Variáveis } & \multirow{2}{*}{$\begin{array}{l}\text { PAID } \\
\text { Total }\end{array}$} & \multicolumn{4}{|c|}{ PAID Subdimensões } \\
\hline & & Emocional & Tratamento & Alimentação & Apoio Social \\
\hline \multicolumn{6}{|l|}{ Sexo } \\
\hline Masculino & 30,76 & 20,22 & 4,31 & 4,51 & 1,70 \\
\hline Feminino & 35,60 & 23,21 & 4,55 & 5,77 & 2,06 \\
\hline \multicolumn{6}{|l|}{ Parceiro } \\
\hline Sim & 34,91 & 22,73 & 4,72 & 5,43 & 2,01 \\
\hline Não & 30,62 & 20,15 & 3,87 & 4,85 & 1,75 \\
\hline \multicolumn{6}{|l|}{ Mora só } \\
\hline Sim & 30,65 & 20,27 & 4,51 & 4,67 & 1,19 \\
\hline Não & 34,22 & 22,30 & 4,50 & 5,36 & $2,04^{*}$ \\
\hline \multicolumn{6}{|c|}{ Antidepressivo } \\
\hline Sim & 42,75 & 28,46 & 5,18 & 6,46 & 2,62 \\
\hline Não & $31,42^{*}$ & $20,38^{*}$ & 4,31 & 4,98 & $1,74^{*}$ \\
\hline \multicolumn{6}{|l|}{ Insulina } \\
\hline Sim & 37,74 & 25,31 & 4,26 & 6,10 & 2,05 \\
\hline Não & 32,21 & 20,82 & 4,53 & 4,97 & 1,87 \\
\hline \multicolumn{6}{|l|}{ Tabagismo } \\
\hline Sim & 30,36 & 20,26 & 3,80 & 4,63 & 1,66 \\
\hline Não & 34,12 & 22,24 & 4,56 & 5,35 & 1,95 \\
\hline
\end{tabular}

*Teste de Mann-Whittney, $\mathrm{p}<0,05$.

A correlação entre o escore de PAID total e PAID emocional com a idade foi, respectivamente, $-0,21$ $(p<0,01)$ e $-0,2306(p<0,01)$. Os escores de PAID total e suas subdimensões não se mostraram associados ao tempo de doença e ao tempo de insulinoterapia.

A Tabela 3 apresenta a análise univariada do sofrimento emocional grave e as variáveis do estudo, e a Tabela 4 apresenta o modelo de regressão final.

\section{Discussão}

Os escores obtidos nesse estudo apontam para valores elevados de sofrimento emocional grave em mais de um terço da amostra estudada, que se mostrou associado a pessoas mais jovens, com mais tempo de doença e que fazem uso de medicação antidepressiva.

O valor médio de SMED aferido nesse estudo se mostrou acima dos valores obtidos em outros estudos nacionais. ${ }^{14-16,21}$ A correlação negativa observada com a idade, ainda que fraca, também se mostrou consistente com esses estudos nacionais. Por outro lado, chama atenção que os valores médios de SMED na dimensão apoio social se mostraram mais altos entre aqueles que moravam com outras pessoas e que tinham parceiro (essa última associação sem significância estatística). Esse achado, aparentemente paradoxal, pode sugerir que ter parceiro ou morar com alguém não garante um apoio social percebido adequado, que pode se revestir mais em uma "cobrança de atitudes" do que uma busca de empoderamento para o autocuidado. ${ }^{23}$

Ainda com relação aos valores médios de SMED, estudos internacionais têm mostrado valores um pouco inferiores ao observado em Blumenau (Espanha, 28,4, Austrália, 26,3 e Suécia 26,6). ${ }^{6,24,25}$ 
Tabela 3. Análise univariada do sofrimento emocional grave e variáveis de estudo.

\begin{tabular}{|c|c|c|c|c|}
\hline \multirow{2}{*}{ Variáveis } & \multicolumn{2}{|c|}{ Sofrimento } & \multirow{2}{*}{ OR (IC 95\%) } & \multirow{2}{*}{$\mathbf{p}$} \\
\hline & $\operatorname{Sim}(\%)$ & Não (\%) & & \\
\hline \multicolumn{5}{|l|}{ Sexo } \\
\hline Masculino & $23(28,1)$ & $59(72,0)$ & & \\
\hline Feminino & $48(42,1)$ & $66(57,9)$ & $1,9(1,0-3,4)$ & $0,045^{\star}$ \\
\hline \multicolumn{5}{|l|}{ Faixa etária } \\
\hline 19-64 anos & $47(39,2)$ & $73(60,8)$ & & \\
\hline 65 e mais & $24(31,2)$ & $52(68,4)$ & $1,6(0,9-3,0)$ & 0,104 \\
\hline \multicolumn{5}{|l|}{ Parceiro } \\
\hline Sim & $53(37,1)$ & $90(62,9)$ & & \\
\hline Não & $18(36,0)$ & $32(64,0)$ & $1,1(0,5-2,0)$ & 0,893 \\
\hline \multicolumn{5}{|l|}{ Mora só } \\
\hline Sim & $8(34,8)$ & $15(65,2)$ & & \\
\hline Não & $63(37,1)$ & $107(62,9)$ & $0,9(0,4-2,3)$ & 0,832 \\
\hline \multicolumn{5}{|l|}{ Escolaridade } \\
\hline Ensino superior, mestrado ou doutorado & $11(29,7)$ & $26(70,1)$ & & \\
\hline Ensino fundamental completo ou incompleto & $56(37,3)$ & $94(62,7)$ & $1,4(0,6-3,1)$ & 0,389 \\
\hline Não alfabetizado ou sem declaração & $4(50,0)$ & $4(50,0)$ & $2,4(0,5-11,1)$ & 0,278 \\
\hline \multicolumn{5}{|l|}{ Uso de Antidepressivos } \\
\hline Não & $50(32,5)$ & $104(67,5)$ & & \\
\hline Sim & $21(52,5)$ & $19(47,5)$ & $2,3(1,1-4,7)$ & $0,021^{*}$ \\
\hline \multicolumn{5}{|l|}{ Tempo de doença } \\
\hline $0-1,9$ anos & $2(12,5)$ & $14(87,5)$ & & \\
\hline $2-4,9$ anos & $20(43,5)$ & $26(56,5)$ & $5,4(1,1-26,5)$ & $0,038^{*}$ \\
\hline 5 anos ou mais & $49(37,1)$ & $83(62,9)$ & $4,1(0,9-19,0)$ & 0,068 \\
\hline \multicolumn{5}{|l|}{ Insulinoterapia } \\
\hline Não & $47(32,6)$ & $97(67,4)$ & & \\
\hline Sim & $24(47,1)$ & $27(52,9)$ & $1,8(1,0-3,5)$ & 0,066 \\
\hline \multicolumn{5}{|l|}{ Tempo de Insulinoterapia } \\
\hline 0 & $47(32,6)$ & $97(67,4)$ & & \\
\hline $0,1-2$ & $4(44,4)$ & $5(55,6)$ & $1,7(0,4-6,4)$ & 0,470 \\
\hline $2 \mathrm{e}$ mais anos & $19(46,3)$ & $22(53,7)$ & $1,8(0,9-3,6)$ & 0,108 \\
\hline \multicolumn{5}{|l|}{ Tabagismo } \\
\hline Sim & $64(37,4)$ & $107(62,6)$ & & \\
\hline Não & $7(29,2)$ & $17(70,8)$ & $0,7(0,3-1,8)$ & 0,496 \\
\hline
\end{tabular}

*Teste de Qui-quadrado $p<0,05$.

Tabela 4. Análise univariada e multivariada por regressão logística não condicional do sofrimento emocional grave e variáveis de estudo (ajustado por sexo e faixa etária) $n=193$.

\begin{tabular}{|c|c|c|c|c|}
\hline & \multicolumn{2}{|c|}{ Análise Univariada } & \multicolumn{2}{|c|}{ Análise multivariada } \\
\hline & OR bruto & $\mathbf{p}$ & OR ajustado & $\mathbf{p}$ \\
\hline \multicolumn{5}{|l|}{ Sexo } \\
\hline Masculino & 1 & & 1 & \\
\hline Feminino & $1,9(1,0-3,4)$ & 0,043 & $1,8(0,9-3,5)$ & 0,071 \\
\hline \multicolumn{5}{|l|}{ Faixa etária } \\
\hline 65 e mais & 1 & & 1 & \\
\hline 19 a 64 anos & $1,6(0,9-3,0)$ & 0,100 & $2,1(1,1-4,1)$ & 0,031 \\
\hline \multicolumn{5}{|c|}{ Tempo de doença (anos) } \\
\hline $0-12$ & 1 & & 1 & \\
\hline $2-15$ & $5,4(1,1-26,5)$ & 0,038 & $6,4(1,1-36,1)$ & 0,035 \\
\hline 5 e mais & $4,1(0,9-19,0)$ & 0,068 & $5,4(1,1-28,8)$ & 0,048 \\
\hline \multicolumn{5}{|c|}{ Uso de antidepressivos } \\
\hline Não & 1 & & 1 & \\
\hline Sim & $2,3(1,1-4,7)$ & 0,021 & $2,8(1,3-6,0)$ & 0,010 \\
\hline
\end{tabular}

1. Razão de Máxima Verossimilhança $=19,62$ 
Nesses países, as pessoas têm maior apoio social e sistemas de saúde historicamente mais estruturados. Além disso, o fato de viverem em um contexto cultural que lida com o processo saúde-doença de maneira diferente pode explicar, ao menos em parte, o pior resultado observado aqui, o que deverá ser investigado em pesquisas futuras.

Nesse estudo, 36,2\% das pessoas apresentaram escore do PAID total maior que 40. Esse resultado se mostrou igual ao resultado sintético de em uma metanálise com 55 estudos provenientes dos 5 continentes com perfil de pacientes semelhante ao nosso. Além disso, nessa metanálise observou-se que o sexo feminino e a presença de depressão se mostraram associados a piores escores. ${ }^{9}$

No presente estudo, pessoas em uso de medicamentos antidepressivos têm um risco 2,3 vezes maior de apresentarem sofrimento emocional grave. O distress associado ao diabetes e a depressão são quadros distintos, englobados no sofrimento emocional, que podem se manifestar associados, e esta associação parece potencializar seus efeitos negativos sobre o controle glicêmico. ${ }^{26,27}$ De qualquer maneira, ambas as condições devem ser diferenciadas, adequadamente tratadas e acompanhadas pelos profissionais da área da saúde. ${ }^{28,29}$

Com relação à faixa etária, nossos resultados indicaram que quanto mais jovem, maior a frequência de sofrimento mental grave. Resultados semelhantes foram obtidos em outros estudos. ${ }^{21,30-33}$ Dentre as dificuldades com o autocuidado do diabetes por jovens adultos, estariam: dificuldades da administração do tempo, estresse, problemas financeiros, agenda irregular, hipoglicemia, restrições, questões de peso e imagem corporal. ${ }^{34}$

O maior tempo de diagnóstico de diabetes se mostrou associado ao sofrimento emocional grave. Isso pode ser explicado, ao menos em parte, pela maior vivência das alterações de estilo de vida, das restrições e dos sintomas impostos pela doença, em relação àquelas pessoas com menor tempo de diagnóstico. Este resultado está de acordo com aqueles de Bernini et al., ${ }^{35}$ que encontraram correlação positiva entre os resultados do B-PAID, cujos valores mais altos indicam mais comprometimentos emocionais com o tempo de diagnóstico de diabetes.

Apesar de não apresentar significância estatística, o sexo feminino teve maiores índices de sofrimento relacionado ao diabetes do que o masculino, resultado consistente com diversos estudos.9,12,13,21 Isso pode ser explicado, ao menos em parte, devido às demandas crescentes da doença associadas aos papéis impostos pela sociedade às mulheres. Espera-se que o sexo feminino assuma papéis importantes tanto na família como na sociedade, tornando as demandas da doença mais desafiadoras para as mulheres. ${ }^{9}$ Por outro lado, os papéis assumidos pelos homens, relacionados à força e provimento do sustento do lar, fazem que os mesmos não admitam a presença de sofrimento emocional, mesmo na presença de doença física. ${ }^{36}$ Ainda que esses papéis se encontrem em constante mudança nas últimas décadas, ainda possuem força em pessoas com mais idade.

Pessoas em insulinoterapia apresentaram razão de chances 1,8 vezes maior de desenvolverem sofrimento mental grave, diferença que não foi significante, mas foi muito próxima. A insulinoterapia contribuiria para o sofrimento mental grave pela preocupação com a administração de insulina, crises hipoglicêmicas e a sensação de fracasso pessoal no controle glicêmico. ${ }^{30,31,33,37}$

Com relação ao nível de escolaridade, os resultados sugerem que, quanto menor for o grau de instrução do indivíduo, maior será a chance de desenvolver sofrimento mental. Esse resultado é compreensível, já que um baixo grau de escolaridade sugere menor capacidade de lidar com as tarefas implicadas na gestão do diabetes. ${ }^{38} \mathrm{O}$ estudo atual não mostrou associação entre os níveis de escolaridade e a presença de sofimento emocional. 
Diante desses resultados, duas questões se colocam para as equipes de saúde: a detecção precoce do sofrimento emocional relacionado ao diabetes, e as estratégias para manejá-lo. Apesar da detecção precoce parecer intuitivamente interessante, em razão da existência de questionários de fácil aplicação e recomendação por autores, ${ }^{39}$ estudo de revisão baseado em ensaios clínicos randomizados sugere que a detecção precoce através de questionários autoaplicáveis não parece ter efeitos benéficos sobre o controle do diabetes. ${ }^{40} \mathrm{Ou}$ seja, recomenda-se que as equipes de ESF invistam em uma abordagem individualizada sobre a pessoa com diabetes a respeito de suas dúvidas e angústias relativas à doença e seu tratamento ao longo do processo de cuidado, ao invés de utilizar estratégias de "screening" para todas as pessoas assistidas no serviço. ${ }^{17,20}$

Por outro lado, intervenções clínicas específicas voltadas para pessoas com SMED ainda precisam ser melhor estudadas. Uma revisão sistemática com metanálise que incluiu 41 ensaios clínicos randomizados $(n=6650)$ encontrou algumas abordagens eficazes para reduzir o sofrimento emocional, ainda que com pequenos efeitos. A abordagem de cuidado individualizado realizada pelo médico generalista da atenção primária teve resultados melhores que aquela feita pelo endocrinologista. Isso pode ser explicado, ao menos em parte, pelo maior acesso e a continuidade oferecidos pela atenção primária. O tempo e número de sessões também refletiram na eficácia, sendo necessárias no mínimo 6 sessões com duração de 13 semanas. Entre as abordagens testadas, as psicopedagógicas e entrevistas motivacionais mostraram melhores resultados. Essas abordagens tendem a estimular maior autonomia dos pacientes em relação ao manejo da diabetes e do sofrimento emocional. ${ }^{41}$

Esse estudo apresenta algumas limitações. Primeiramente, trata-se de um estudo transversal, o que limita a compreensão causal com base no modelo ajustado obtido. Outra preocupação se refere às características amostrais. Nosso estudo optou por uma amostragem intencional de unidades de saúde, não tendo assim a pretensão de representar a totalidade das unidades de Blumenau. Além disso, teve perdas e recusas de 19,7\%. Contudo, as características sociodemográficas e clínicas da amostra se mostraram consistentes com outras pesquisas realizadas na atenção primária, com predomínio do sexo feminino, com DM2, mais de 40 anos e baixo grau de escolaridade. ${ }^{42} \mathrm{Em}$ outros estudos nacionais a amostra também possuía características sociodemográficas semelhantes às apresentadas pelos sujeitos deste estudo. Por fim, o tamanho amostral obtido diminuiu o poder estatístico. No entanto, os resultados das medidas de efeito se mostraram consistentes com a literatura.

\section{Conclusões}

Os resultados do estudo sugerem que cerca de um terço das pessoas com diabetes na atenção primária apresentam SMED. Essa condição se mostrou associada a mulheres, mais jovens, com mais de 2 anos de diagnóstico da doença e em uso de medicação antidepressiva. Pessoas com esse perfil devem ter seu cuidado priorizado pelas equipes de saúde na atenção primária.

Outros estudos nessa área se fazem necessários, em particular com delineamento longitudinal, para elucidar questões relativas à incidência, possíveis fatores causais e o papel prognóstico do SMED nas complicações de pessoas com diabetes assistidas na atenção primária. 


\title{
Conflito de interesses
}

\author{
Declaram não haver.
}

\section{Referências}

1. Iser BPM, Stopa SR, Chueiri PS, Szwarcwald CL, Malta DC, Monteiro HOC, et al. Prevalência de diabetes autorreferido no Brasil: resultados da Pesquisa Nacional de Saúde 2013. Epidemiol Serv Saúde. 2015;24(2):305-14. https://doi.org/10.5123/S1679-49742015000200013

2. Ministério da Saúde (BR), Secretaria de Vigilância em Saúde, Departamento de Vigilância de Doenças e Agravos não Transmissíveis e Promoção da Saúde. VIGITEL Brasil 2013: Vigilância de Fatores de Risco e Proteção para Doenças Crônicas por Inquérito Telefônico. Brasília (DF): Ministério da Saúde; 2014.

3. Welch GW, Jacobson AM, Polonsky WH. The Problem Areas in Diabetes Scale. An evaluation of its clinical utility. Diabetes Care. 1997;20(5):760-6. https://doi.org/10.2337/diacare.20.5.760

4. Polonsky WH, Anderson BJ, Lohrer PA, Welch G, Jacobson AM, Aponte JE, et al. Assessment of diabetes-related distress. Diabetes Care. 1995;18(6):754-60. https://doi.org/10.2337/diacare.18.6.754

5. Co MA, Tan LS, Tai ES, Griva K, Amir M, Chong KJ, et al. Factors associated with psychological distress, behavioral impact and health-related quality of life among patients with type 2 diabetes mellitus. J Diabetes Complications. 2015;29(3):378-83. https://doi.org/10.1016/j.jdiacomp.2015.01.009

6. Reddy J, Wilhelm K, Campbell L. Putting PAID to diabetes-related distress: the potential utility of the problem areas in diabetes (PAID) scale in patients with diabetes. Psychosomatics. 2013;54(1):44-51. https://doi.org/10.1016/j.psym.2012.08.004

7. Pouwer F, Beekman AT, Lubach C, Snoek FJ. Nurses' recognition and registration of depression, anxiety and diabetes-specific emotional problems in outpatients with diabetes mellitus. Patient Educ Couns. 2006;60(2):235-40. https://doi.org/10.1016/j.pec.2005.01.009

8. Jimenez-Garcıa R, Martinez Huedo MA, Hernandez-Barrera V, Lopez de Andres A, Martinez D, Jimenez-Trujillo I, et al. Psychological distress and mental disorders among Spanish diabetic adults: a case-control study. Prim Care Diabetes. 2012;6(2):149-56. https://doi. org/10.1016/j.pcd.2011.10.002

9. Perrin NE, Davies MJ, Robertson N, Snoek FJ, Khunti K. The prevalence of diabetes-specific emotional distress in people with Type 2 diabetes: a systematic review and meta-analysis. Diabet Med. 2017;34(11):1508-20. https://doi.org/10.1111/dme.13448

10. Tan LS, Khoo EY, Tan CS, Griva K, Mohamed A, New M, et al. Sensitivity of three widely used questionnaires for measuring psychological distress among patients with type 2 diabetes mellitus. Qual Life Res. 2015;24(1):153-62. https://doi.org/10.1007/s11136-014-0747-z

11. Graue M, Haugstvedt A, Wentzel-Larsen T, Iversen MM, Karlsen B, Rokne B. Diabetes-related emotional distress in adults: reliability and validity of the Norwegian versions of the Problem Areas in Diabetes Scale (PAID) and the Diabetes Distress Scale (DDS). Int J Nurs Stud. 2012;49(2):174-82. https://doi.org/10.1016/j.ijnurstu.2011.08.007

12. Snoek FJ, Pouwer F, Welch GW, Polonsky WH. Diabetes-related emotional distress in Dutch and U.S. diabetic patients: cross-cultural validity of the problem areas in diabetes scale. Diabetes Care. 2000;23(9):1305-9. https://doi.org/10.2337/diacare.23.9.1305

13. Papathanasiou A, Koutsovasilis A, Shea S, Philalithis A, Papavasiliou S, Melidonis A, et al. The Problem Areas in Diabetes (PAID) scale: psychometric evaluation survey in a Greek sample with type 2 diabetes. J Psychiatr Ment Health Nurs. 2014;21(4):345-53. https://doi.org/10.1111/j.1365-2850.2012.01875.x

14. Gross CC, Gross JL, Goldim JR. Problemas emocionais e percepção de coerção em pacientes com diabetes tipo 2: um estudo observacional. Rev HCPA. 2010;30(4):431-5.

15. Zanchetta FC, Trevisan DD, Apolinario PP, Silva JB, Lima MH. Clinical and sociodemographic variables associated with diabetes-related distress in patients with type 2 diabetes mellitus. Einstein (São Paulo). 2016;14(3):346-51. https://doi.org/10.1590/S1679-45082016AO3709

16. Souza ECS, Souza SA, Alves TOS, Gois CFL, Guimarães AMDN, Mattos MCT, et al. Avaliação da qualidade de vida de portadores de diabetes utilizando a medida específica B-PAID. Rev Min Enferm. 2012;16(4):509-15.

17. Ministério da Saúde (BR), Secretaria de Atenção à Saúde, Departamento de Atenção Básica. Estratégias para o Cuidado da Pessoa com Doença Crônica: Diabetes Mellitus. Brasília (DF): Ministério da Saúde; 2013.

18. Chediek J, Mulleady AI, Soares S, Marilena C. Atlas do Desenvolvimento Humano - Perfil [Internet]. Brasília (DF): Fundação João Pinheiro, IPEA, PNUD; 2014. Disponível em: http://www.atlasbrasil.org.br/2013/pt/perfil_m/blumenau_sc 
19. Ministério da Saúde (BR), Departamento de Informática do SUS (DATASUS). HIPERDIA - Sistema de Cadastramento e Acompanhamento de Hipertensos e Diabéticos. 2016 [Internet]. 2016 [acesso 2016 Maio 18]. Disponível em: http://datasus.saude.gov.br/sistemas-eaplicativos/epidemiologicos/hiperdia

20. Oliveira JEP, Vencio S, orgs. Diretrizes da Sociedade Brasileira de Diabetes 2015-2016. São Paulo: AC Farmacêutica; 2016.

21. Gross CC, Scain SF, Scheffel R, Gross JL, Hutz CS. Brazilian version of the Problem Areas in Diabetes Scale (B-PAID): validation and identification of individuals at high risk for emotional distress. Diabetes Res Clin Pract. 2007;76(3):455-9. https://doi.org/10.1016/j. diabres.2006.09.022

22. Hermanns N, Kulzer B, Krichbaum M, Kubiak T, Haak T. How to screen for depression and emotional problems in patients with diabetes: Comparison of screening characteristics of depression questionnaires, measurement of diabetes-specific emotional problems and standard clinical assessment. Diabetologia. 2006;49(3):469-77. https://doi.org/10.1007/s00125-005-0094-2

23. Cyrino AP, Schraiber LB, Teixeira RR. Education for type 2 diabetes mellitus self-care: from compliance to empowerment. Interface (Botucatu). 2009;13(30):93-106. https://doi.org/10.1590/S1414-32832009000300009

24. Beléndez M, Hernández-Mijares A, Marco J, Domínguez JR, Pomares FJ. Validation of the Spanish version of the Problem Areas in Diabetes (PAID-SP) Scale. Diabetes Res Clin Pract. 2014;106(3):e93-5. https://doi.org/10.1016/j.diabres.2014.09.012

25. Amsberg S, Wredling R, Lins PE, Adamson U, Johansson UB. The psychometric properties of the Swedish version of the Problem Areas in Diabetes Scale (Swe-PAID-20): Scale development. Int J Nurs Stud. 2008;45(9):1319-28. https://doi.org/10.1016/j.jijnurstu.2007.09.010

26. Schmitt A, Reimer A, Kulzer B, Haak T, Gahr A, Hermanns N. Negative association between depression and diabetes control only when accompanied by diabetes-specific distress. J Behav Med. 2015;38(3):556-64. https://doi.org/10.1007/s10865-014-9604-3

27. Kreider KE. Diabetes Distress or Major Depressive Disorder? A Practical Approach to Diagnosing and Treating Psychological Comorbidities of Diabetes. Diabetes Ther. 2017;8(1):1-7. https://doi.org/10.1007/s13300-017-0231-1

28. Fisher L, Gonzalez JS, Polonsky WH. The confusing tale of depression and distress in patients with diabetes: a call for greater clarity and precision. Diabet Med. 2014;31(7):764-72. https://doi.org/10.1111/dme.12428

29. Snoek FJ, Bremmer MA, Hermanns N. Constructs of depression and distress in diabetes: time for an appraisal. Lancet Diabetes Endocrinol. 2015;3(6):450-60. https://doi.org/10.1016/S2213-8587(15)00135-7

30. Karlsen B, Oftedal B, Bru E. The relationship between clinical indicators, coping styles, perceived support and diabetes-related distress among adults with type 2 diabetes. J Adv Nurs. 2012;68(2):391-401. https://doi.org/10.1111/j.1365-2648.2011.05751.x

31. Delahanty LM, Grant RW, Wittenberg E, Bosch JL, Wexler DJ, Cagliero E, et al. Association of diabetes-related emotional distress with diabetes treatment in primary care patients with Type 2 diabetes. Diabet Med. 2007;24(1):48-54. https://doi.org/10.1111/j.14645491.2007.02028.x

32.Pintaudi B, Lucisano G, Gentile S, Bulotta A, Skovlund SE, Vespasiani G, et al.; BENCH-D Study Group. Correlates of diabetes-related distress in type 2 diabetes: Findings from the benchmarking network for clinical and humanistic outcomes in diabetes (BENCH-D) study. J Psychosom Res. 2015;79(5):348-54. https://doi.org/10.1016/j.jpsychores.2015.08.010

33. Stoop CH, Nefs G, Pop VJ, Wijnands-van Gent CJM, Tack CJ, Geelhoed-Duijvestijn PH, et al. Diabetes-specific emotional distress in people with Type 2 diabetes: a comparison between primary and secondary care. Diabet Med. 2014;31(10):1252-9. https://doi.org/10.1111/ dme.12472

34. Garvey KC, Wolpert HA. Identifying the unique needs of transition care for young adults with type 1 diabetes. Diabetes Spectr.2011;24(1):225. https://doi.org/10.2337/diaspect.24.1.22

35. Bernini LS, Barrile SR, Mangili AF, Arca EA, Correr R, Ximenes MA, et al. O impacto do diabetes mellitus na qualidade de vida de pacientes da Unidade Básica de Saúde. Cad Bras Ter Ocup. 2017;25(3):533-41. https://doi.org/10.4322/2526-8910.ctoAO0899

36. Figueiredo WS, Schraiber LB. Concepções de gênero de homens usuários e profissionais de saúde de serviços de atenção primária e os possíveis impactos na saúde da população masculina, São Paulo, Brasil. Ciênc Saúde Coletiva. 2011;16(Suppl 1):935-44. https://doi.org/10.1590/S1413-81232011000700025

37. Polonsky WH, Fisher L, Guzman S, Villa-Caballero L, Edelman SV. Psychological insulin resistance in patients with type 2 diabetes: The scope of the problem. Diabetes Care. 2005;28(10):2543-5. https://doi.org/10.2337/diacare.28.10.2543

38. Spencer MS, Kieffer EC, Sinco BR, Palmisano G, Guzman JR, James SA, et al. Diabetes-Specific Emotional Distress among African Americans and Hispanics with Type 2 Diabetes. J Health Care Poor Underserved. 2006;17(2 Suppl):88-105. https://doi.org/10.1353/ hpu.2006.0095 
39. Beverly EA, Ivanov NN, Court AB, Fredricks TR. Is diabetes distress on your radar screen? J Fam Pract. 2017;66(1):9-14.

40. Pouwer F. Should we screen for emotional distress in type 2 diabetes mellitus? Nat Rev Endocrinol. Nat Rev Endocrinol. 2009;5(12):665-71. https://doi.org/10.1038/nrendo.2009.214

41. Sturt J, Dennick K, Hessler D, Hunter BM, Oliver J, Fisher L. Effective interventions for reducing diabetes distress: systematic review and meta-analysis. Int Diabetes Nurs. 2015;12(2):40-55. https://doi.org/10.1179/2057332415Y.0000000004

42. Ferreira CLRA, Ferreira MG. Características epidemiológicas de pacientes diabéticos da rede pública de saúde - análise a partir do sistema HiperDia. Arq Bras Endocrinol Metabol. 2009;53(1):80-6. https://doi.org/10.1590/S0004-27302009000100012 